\title{
Psychophysical Evaluation of a Variable Friction Tactile Interface
}

\author{
Evren Samur ${ }^{\mathrm{a}}$, J. Edward Colgate ${ }^{\mathrm{b}}$, and Michael A. Peshkin ${ }^{\mathrm{b}}$ \\ ${ }^{a}$ Robotic Systems Laboratory, Ecole Polytechnique Fédérale de Lausanne, Switzerland; \\ ${ }^{\mathrm{b}}$ Laboratory for Intelligent Mechanical Systems, Northwestern University, IL USA
}

\begin{abstract}
This study explores the haptic rendering capabilities of a variable friction tactile interface through psychophysical experiments. In order to obtain a deeper understanding of the sensory resolution associated with the Tactile Pattern Display $(\mathrm{TPaD})$, friction discrimination experiments are conducted. During the experiments, subjects are asked to explore the glass surface of the TPaD using their bare index fingers, to feel the friction on the surface, and to compare the slipperiness of two stimuli, displayed in sequential order. The fingertip position data is collected by an infrared frame and normal and translational forces applied by the finger are measured by force sensors attached to the TPaD. The recorded data is used to calculate the coefficient of friction between the fingertip and the TPaD. The experiments determine the just noticeable difference (JND) of friction coefficient for humans interacting with the TPaD.
\end{abstract}

Keywords: Psychophysical evaluation, tactile feedback, friction reduction, just noticeable difference

\section{INTRODUCTION}

Our work lies is in the realm of haptics for human-computer interface. Although haptics is not generally considered a strong pathway for communication of structured information such as text, it is in fact an extremely rich, multi-modal pathway. Some evidence of this lies in the remarkable ability of people to identify common objects quickly and accurately through touch alone [1]. This capability is subserved by a set of stereotyped "Exploratory Procedures" (EPs) such as lateral motion, static contact, pressure, and contour following. The long-range goal of this research is to develop novel interfaces that take advantage of EPs and human tactual identification strategies.

As a first step, we have developed the Tactile Pattern Display (TPaD) [2]. The TPaD employs ultrasonic vibrations to create a squeeze film of air between the vibrating surface and a fingertip, thereby reducing the friction. Similar devices have been developed by Watanabe and Fukui [3], Nara et al. [4], and Biet et al. [5]. Variation in friction level creates shear forces on the fingertip, which are used to present both geometric and material properties of an object through the EP of lateral motion.

This study explores the haptic rendering capabilities of the TPaD through psychophysical experiments. Psychophysics is necessary for several reasons. First, we want to obtain a deeper understanding of the sensory resolution associated with the TPaD. Perhaps because such devices were not previously available, there is a dearth of data describing detection thresholds and subjective magnitude associated with friction and patterns created by variable friction. Second, we want to understand how variations in friction can give rise to the percept of shape. Anecdotal evidence assures us that shape recognition can be driven by a frictional display, but the mechanisms are not understood.

From a perceptual perspective, friction has received very little psychophysical analysis. The lack of systematic work on the perception of friction reflects the difficulty of controlling a range of friction values in physical objects. Virtual environments present a potential solution to this problem, but studies of friction perception with force-feedback devices have not been directed toward an understanding of basic human processes. Notably, these studies required subjects to experience friction forces via a hand-held probe, not via the bare fingertip. The TPaD is unique in that the frictioncontrolled virtual environment is experienced by the bare fingertip.

In this work, we will exploit not only the TPaD's ability to control friction, but its ability to modulate friction spatially and temporally, producing lateral force fields (LFFs). Robles-De-La-Torre and Hayward [6] found that, despite the loss of all proprioceptive and kinesthetic geometric cues, subjects were able to identify virtual bumps and holes given the appropriate lateral force fields. When subjects were given the physical displacement of a bump but played the LFF of a hole, the subjects ignored the geometric cues and identified the object as a hole.

*colgate@northwestern.edu; phone 1-847-491-4264; fax 1-847-491-3915; mech.northwestern.edu/colgate

Human Vision and Electronic Imaging XIV, edited by Bernice E. Rogowitz,

Thrasyvoulos N. Pappas, Proc. of SPIE-IS\&T Electronic Imaging, SPIE Vol. 7240, 72400

(C) 2009 SPIE-IS\&T · CCC code: 0277-786X/09/\$18 · doi: 10.1117/12.817170 


\section{FRICTION DISCRIMINATION EXPERIMENT}

This study explores the haptic rendering capabilities of a Tactile Pattern Display (TPaD) through a friction discrimination experiment. Subjects are asked to explore the display surface with their bare index finger and describe to the researchers the sensations they feel. The friction discrimination experiment determines the just noticeable difference (JND) of friction coefficient for humans interacting with the TPaD.

\subsection{Tactile Pattern Display (TPaD)}

This display consists of two $16 \mathrm{~mm}$ diameter, $0.5 \mathrm{~mm}$ thick piezo ceramic disks (PI Ceramic $\mathrm{GmbH}$ ) glued to the top and bottom of a $50.8 \times 25.4 \times 4.9 \mathrm{~mm}$ glass plate (see Fig.1). The plate is fixed to an aluminum frame by 4 nylon-tip set screws. The disks generate ultrasonic vibrations on the plate when an alternating voltage difference is applied. A high frequency, sinusoidal signal is generated by a signal generator and multiplied by a computer generated analog output to be able to control the amplitude of the excitation voltage. Then, the signal is amplified and applied to the piezo disks.
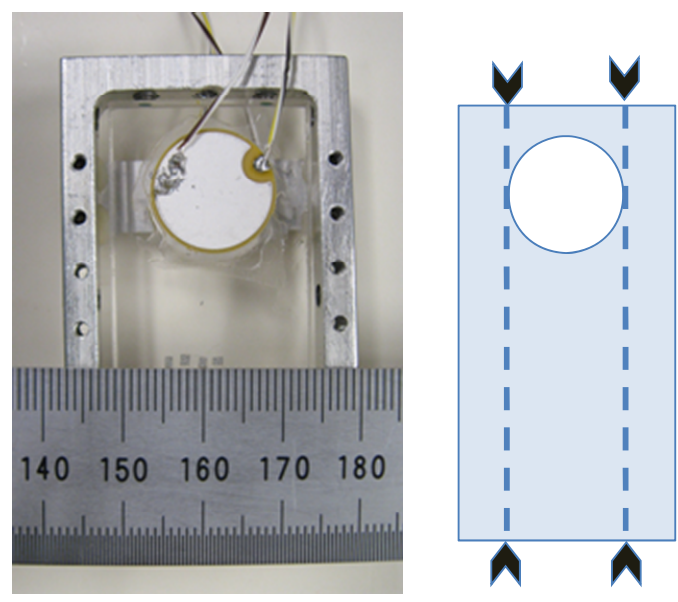

Fig. 1. Rectangular TPaD: Two $16 \mathrm{~mm}$ diameter, $0.5 \mathrm{~mm}$ thick piezo ceramic disks glued to the top and bottom of a $50.8 \mathrm{x}$ $25.4 \times 4.9 \mathrm{~mm}$ glass plate (left). Plate is fixed to the aluminum frame at nodal lines by 4 nylon tip set screws (right).

In order to characterize the frequency behavior of the $\mathrm{TPaD}$, we have performed a dynamic analysis. Velocity of the vibrations was measured using a Laser Doppler Vibrometer (LDV) as the piezo disks were driven with white noise. These data were used to derive a transfer function, which is shown in Fig. 2. A resonant peak is found at $38.5 \mathrm{kHz}$, and this peak has a Q-factor (resonant frequency divided by the peak width at half-height) of 100. Flexural vibrations at this ultrasonic frequency create a squeeze film of air between the plate surface and the finger, thus reducing the friction between the plate and the fingertip as the amplitude of the applied voltage is increased. The mechanisms of friction reduction have been discussed more fully in Biet et al. [7]. We performed a preliminary user study and found that the squeeze film effect between the glass plate and the finger starts to be felt at a vibration amplitude of around 1.5 microns.

\subsection{Experimental Setup}

During the experiments, the subject's finger position was tracked by a commercial infrared (IR) touch screen frame (see Fig. 3). A commercial web camera was also used to record the finger thumb. In addition to the finger tip position recording, normal and translational forces applied by the finger were measured by two 0.25 and $0.5 \mathrm{lb}$ capacity load cells (type: LSM250, FUTEK Advanced Sensor Technology, Inc.) attached to the TPaD device. The normal and friction forces were collected throughout the trial at a sampling rate of $1000 \mathrm{~Hz}$. The finger position was recorded at $100 \mathrm{~Hz}$. 

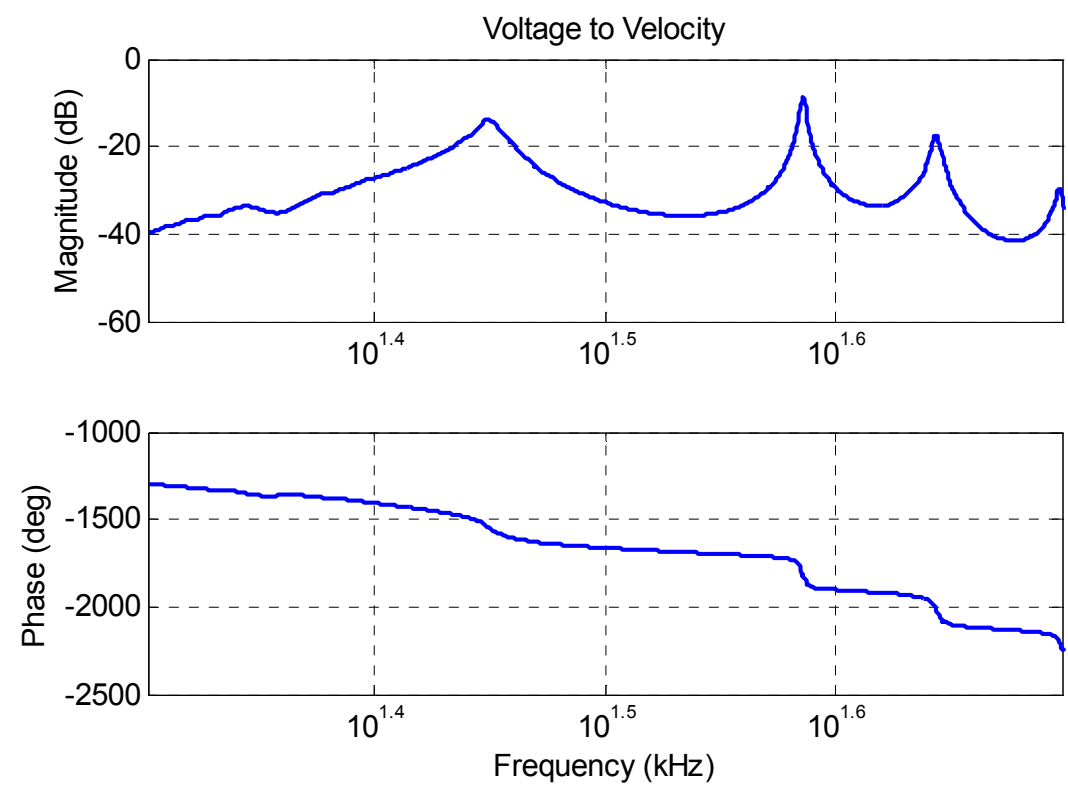

Fig. 2. Transfer function of the TPaD including the amplifier. White noise voltage is given by the signal generator and velocity of vibrations measured by the LDV.
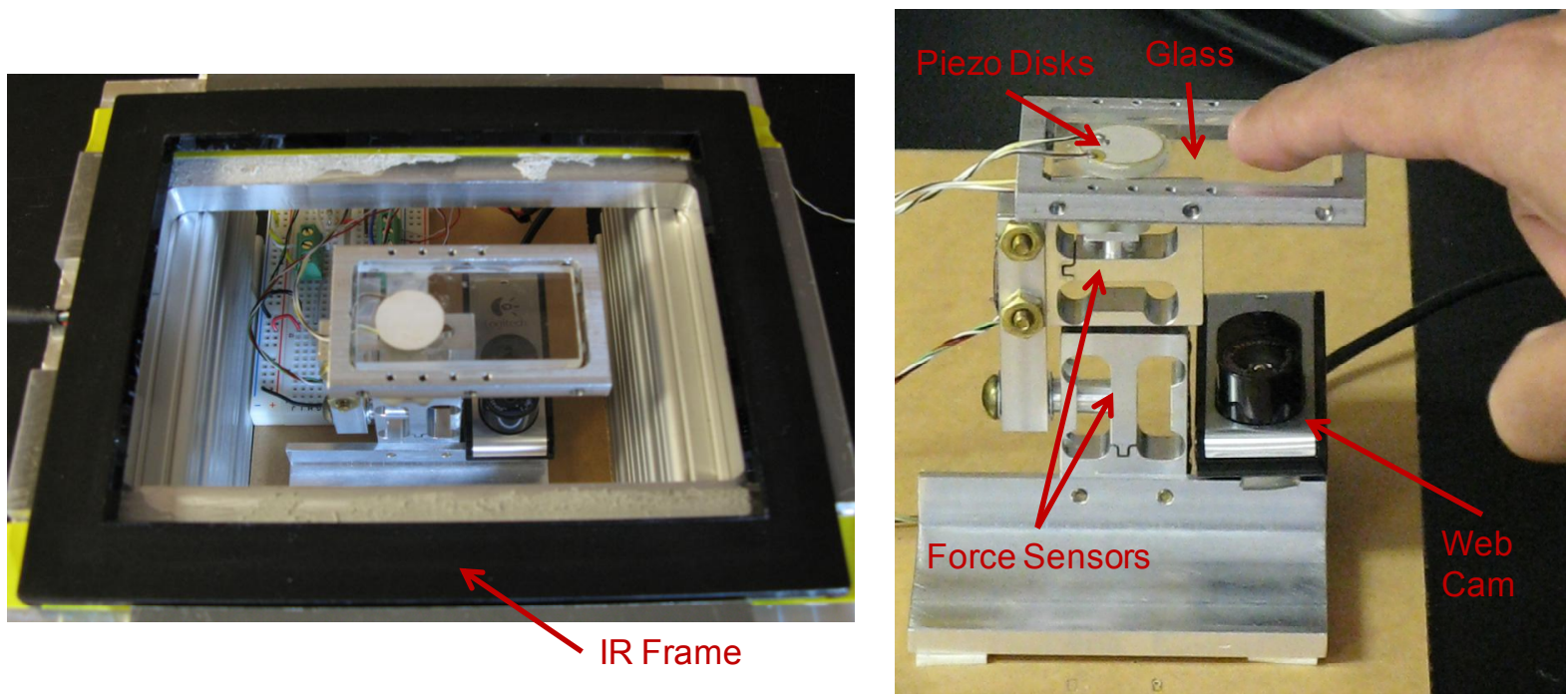

Fig. 3. TPaD and the experimental setup. While a subject is interacting with the TPaD as shown on the right, finger position and normal and friction forces are recorded.

\subsection{Experimental Method}

The friction discrimination experiment determines the just noticeable difference (JND) of friction coefficient for humans interacting with the TPaD. The experiment requires the subject to compare the slipperiness of two stimuli, displayed in sequential order. A baseline value of coefficient of friction is always one of the two stimuli presented in the discrimination task. The other stimulus is a test value to compare against the baseline. The subject is asked to report which stimulus has a higher coefficient of friction. The subject explores two stimuli and chooses the one with higher coefficient of friction. He/she can also choose the answer "same / can't tell". In all experiments, the subject is interacting with the TPaD shown in Fig. 3. 
The first test value is chosen to be $5 \Delta \mathrm{V}$ higher than the baseline. The next trial's test value is adjusted according to Kaernbach's adaptive staircase method [8] until a final JND is found. If the subject correctly identifies which stimulus has a higher coefficient of friction, the excitation voltage of the test stimuli for the next trial is brought closer to the baseline stimulus by $\Delta \mathrm{V}$. If the subject is incorrect, the difference between the baseline and next trial's test stimulus is increased by $3 \Delta \mathrm{V}$. If the subject answers that the stimuli are the "same / can't tell," the difference between the baseline and next trial's test stimulus is increased by $\Delta \mathrm{V}$. These quantities were determined using Kaernbach's formula for a target performance of $75 \%$ correct. The JND is reached when the subject has performed eight reversals within a baseline set. A reversal is when the direction of adjusting $\Delta \mathrm{V}$ changes. The difference threshold for the particular baseline stimulus is evaluated by averaging the test stimuli values between the 4 th and 8 th reversal.

A set of trials takes place for each baseline excitation voltage. Different baseline values are necessary since JND values for friction coefficients might depend on the magnitude of the friction coefficient. The baseline values for excitation voltages (peak-to-peak) on the $\mathrm{TPaD}$ are $0,10,20,30,40,50,60 \mathrm{~V}$ corresponding to the coefficient of friction of 0.95 , $0.7,0.6,0.4,0.3,0.2,0.17$ respectively. The step size, $\Delta \mathrm{V}$, is chosen as $5 \mathrm{~V}$.

During each trial the subject moves his/her finger back and forth on the disk, attempting to maintain a constant normal force and velocity. Throughout each trial the subject is free to toggle between the baseline and the test stimuli as many as he/she wants. The finger is lifted off the plate at each toggle.

\section{RESULTS}

Preliminary data from one subject (the lead author) are presented. A total of 96 data collection trials were performed. An example of data obtained during a trial is shown in Fig.4. The data is from a whole trial with 4 toggles between the baseline stimulus (excitation voltage $0 \mathrm{~V}$, i.e., $\mathrm{TPaD}$ is $\mathrm{OFF}$ ) and the test stimulus ( $10 \mathrm{~V}$ peak-to-peak). The coefficient of friction $(\mu)$ between the finger and the plate surface was calculated using the formula of coulomb friction. The dashed line in the $3^{\text {rd }}$ row of Fig. 4 shows the mean coefficient of friction which is averaged for each stimulus after a threshold is applied. As seen in the figure, the coefficient of friction is around 0.95 when TPaD is OFF and $10 \mathrm{~V}$ peak-to-peak results in 0.25 reduction of the friction coefficient value. The subject responded "different" after exploring the two stimuli. Therefore, we can conclude that $10 \mathrm{~V}$ excitation reduces the friction perceivably.
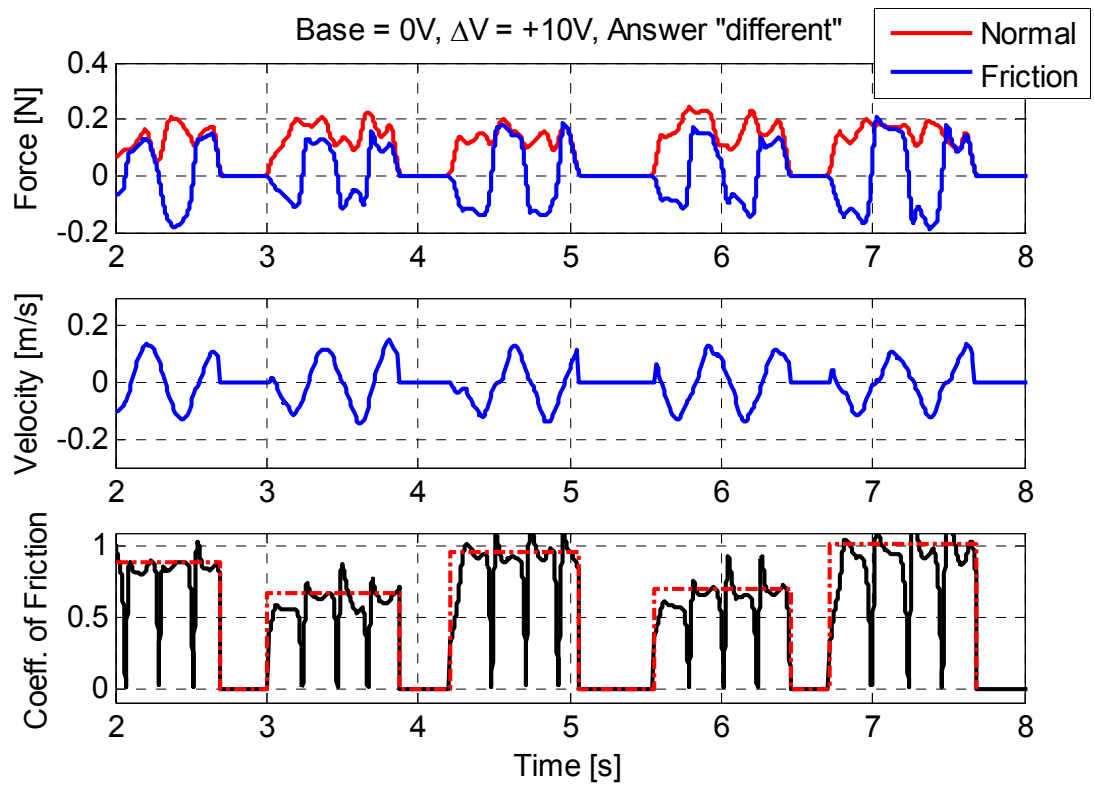

Fig. 4. When the TPaD is OFF, the coefficient of friction is around 0.95 . Even $10 \mathrm{~V}$ peak-to-peak excitation (corresponding to a friction coefficient of $\mu=0.7$ ) creates a perceivable difference. Subject responds "different" and correctly identifies the stimulus with a higher coefficient of friction 
Fig.5 and Fig.6 show two cases in which the subject responded "different" and "same", respectively. In Fig.5, the baseline value of the coefficient of friction is 0.35 (excitation voltage is $30 \mathrm{~V}$ ) and it reduces to 0.2 when $45 \mathrm{~V}$ is applied. The subject perceives the difference of 0.15 in the coefficient of friction at this baseline value. On the other hand, a 0.05 difference in the coefficient of friction is not recognizable with respect to the baseline value of $0.3(40 \mathrm{~V})$ as shown in Fig. 6.
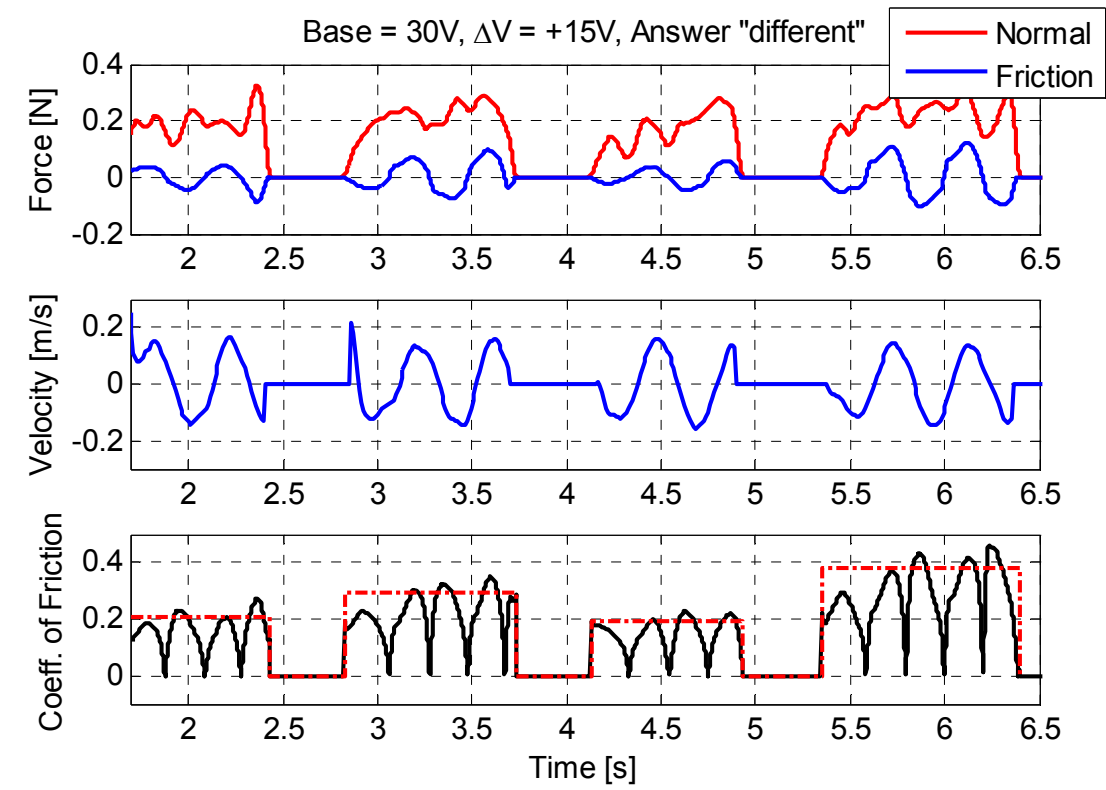

Fig. 5. $30 \mathrm{~V}$ and $45 \mathrm{~V}$ correspond to the coefficients of friction of $\mu=0.35$ and 0.2 , respectively. The subject perceives the difference of 0.15 in the coefficient of friction.
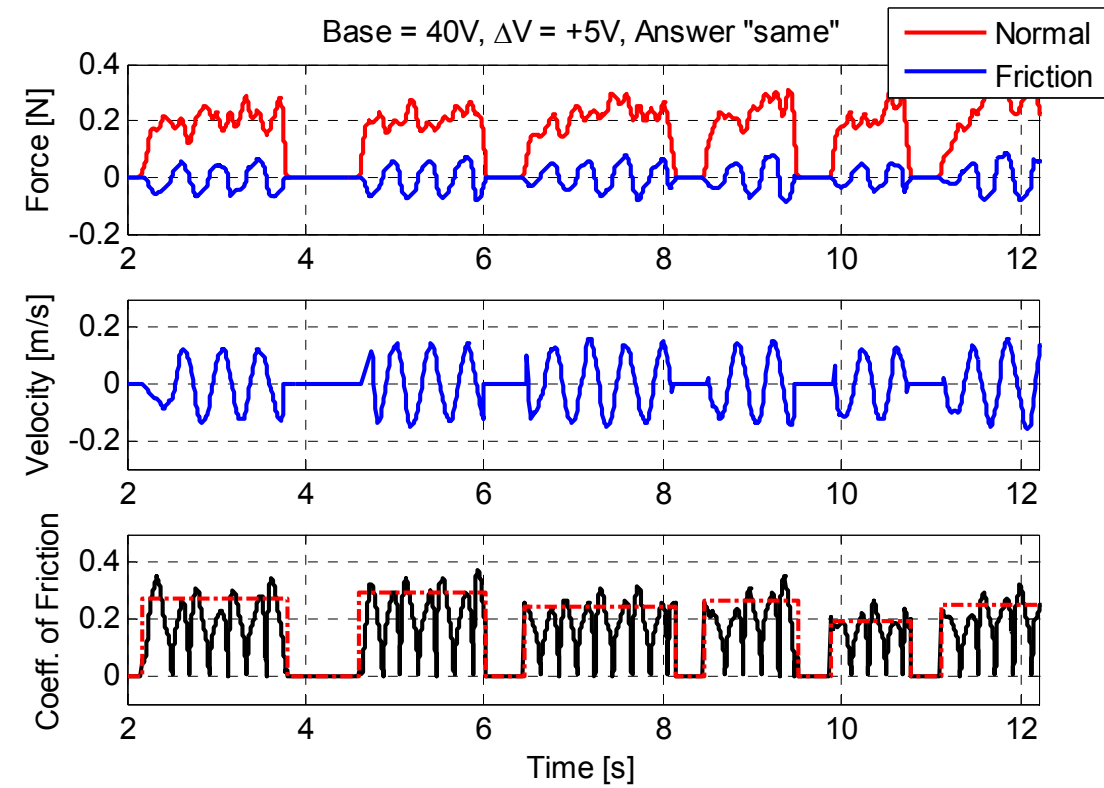

Fig. 6. At the baseline value of $\mu=0.3$ (corresponding to $40 \mathrm{~V}$ ), 0.05 difference in the coefficient of friction ( $5 \mathrm{~V}$ ) is not distinguishable. Subject responds "same". 
The coefficient of friction for each excitation voltage is shown in Fig.7. The data points represent the mean values of the coefficient of friction for each excitation voltage and the vertical bars correspond to the standard deviations in the coefficient of friction over stimuli. As shown in the figure, reduction of friction starts at $10 \mathrm{~V}$ peak-to-peak excitation voltage and reaches a limit above $70 \mathrm{~V}$.

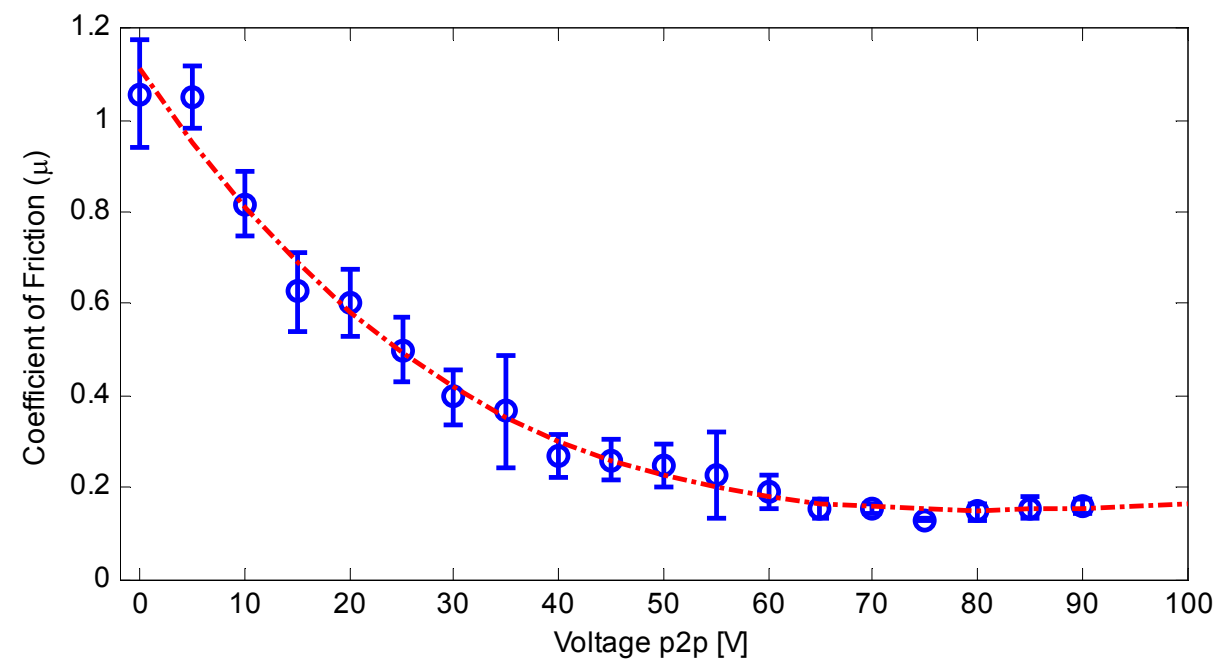

Fig. 7. Reduction of friction as a function of excitation voltage. The data points represent the mean values of the coefficient of friction derived from each stimulus and the vertical bars correspond to the standard deviations over stimuli. The dashed line represents curve fitting to the data points.

\section{CONCLUSIONS AND FUTURE WORK}

The data for this one subject may be summarized as a plot of Weber Fraction versus baseline level, as shown in Fig. 8 . The average JND of friction is $18 \%$. This is similar to that of other haptic modalities such as compliance $(22 \%$ as reported in Tan, et al., [9]), force and viscosity (15\% and 34\%, respectively, as reported in Jones and Hunter, [10]). This result is encouraging, suggesting that friction modulation may be successfully used to present haptic effects to the bare finger, just as compliance and viscosity modulation have been used to present haptic effects to the whole hand. Of course, tests with additional subjects are necessary before firm conclusions can be drawn.

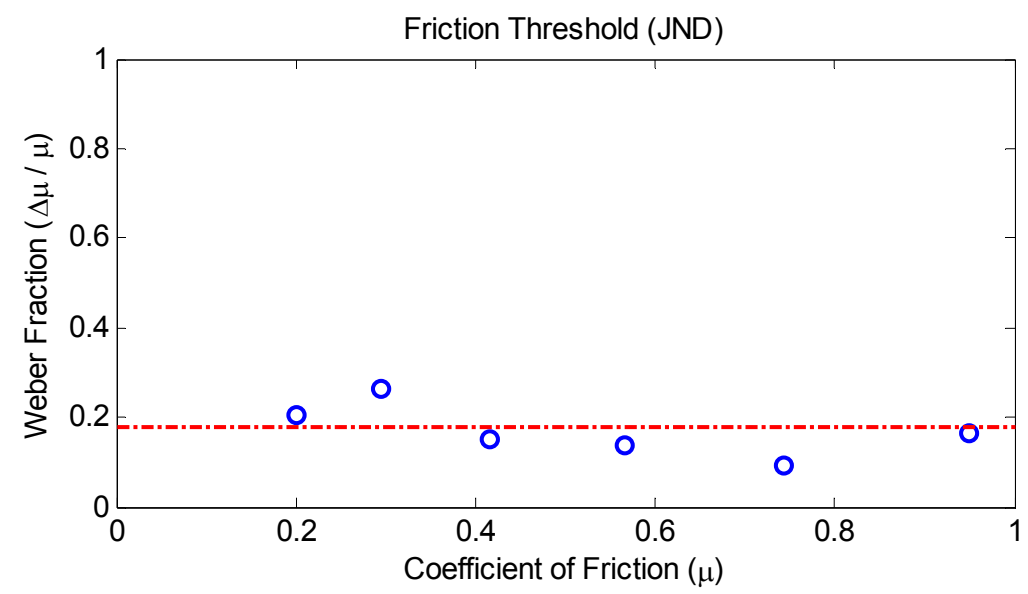

Fig. 8. Just noticeable difference in friction represented as a Weber Fraction and plotted versus baseline coefficient of friction (stimulus intensity). The Weber Fraction appears to be relatively independent of stimulus intensity. 
The recorded data in this experiment (e.g. area of contact, applied tangential and normal forces) can also be used to examine the exploratory behavior of subjects in order to better understand how humans perceive rendered textures.

We are currently carrying out the friction discrimination experiment with additional subjects. In addition to the friction discrimination experiment, we are planning to perform two other psychophysical experiments:

(1) Magnitude Estimation of Friction: This experiment will quantify suprathreshold sensitivity to variations in friction. The ultimate goal is to understand how the physical interaction between skin and surface gives rise to the perception of friction. The subject will explore a surface and give a free numerical rating of its friction intensity. A magnitudeestimation function, relating the mean rated perceptual intensity to the physical dimension, will be obtained. The subject will repeat the same procedure with applying different normal forces and exploring at different finger velocities. We will relate changes in the percept of friction to both the physical variation and the exploratory parameters including speed, force and RMS deviation from a straight line.

(2) Shape Identification: The last experiment will determine the range of shapes that may be conveyed by the TPaD, with respect to spatial extent (size), spatial frequency content (sharpness), and exploratory parameters. This experiment will also quantify the threshold for discriminating a grating from a smooth surface. The subject will explore rendered Gaussian shapes (virtual "bumps" and "holes") and be instructed to locate the highest/lowest point of the highest/deepest perceived bump/hole. Data recorded will include final position of the finger, normal and tangential forces and the subject's shape identification response.

\section{ACKNOWLEDGEMENTS}

The authors would like to acknowledge valuable discussions with Prof. Roberta Klatzky. This work was supported by National Science Foundation Grant No. 0413204 and in part by the NCCR Co-Me of the Swiss National Science Foundation.

\section{REFERENCES}

[1] Lederman, S.J., \& Klatzky, R. L. (1987). Hand movements: A window into haptic object recognition. Cognitive Psychology, 19, 342-368.

[2] Winfield, L., Glassmire, J., Colgate, J., and Peshkin, M. (2007). TPaD: Tactile pattern display through variable friction reduction. Second Joint Eurohaptics Conference and Symposium on Haptic Interfaces for Virtual Environment and Teleoperator Systems, Tsukuba, Japan

[3] Watanabe, T. \& Fukui, S. (1995). A method for controlling tactile sensation of surface roughness using ultrasonic vibration. Proc. IEEE International Conf. on Robotics and Automation, Nagoya, Japan.

[4] Nara, T., Takasaki, M., Maeda, T., Higuchi, T., Ando, S., \& Tachi, S. (2001). Surface acoustic wave tactile display. IEEE Computer Graphics and Applications, 21(6), 56-63.

[5] Biet, M., Giraud, F., Martinot, F. and Semail, B. (2006). A piezoelectric tactile display using traveling lamb wave. Proc. Eurohaptics, Paris, France.

[6] Robles-De-La-Torre, G. and Hayward, V. (2001). Force can overcome geometry in the perception of shape through active touch. Nature, 412, 445-448.

[7] Biet, M., Giraud, F. and Semail, B. (2007). Squeeze film effect for the design of an ultrasonic tactile plate. IEEE Trans. on Ultrasonics, Ferroelectrics, and Frequency Control, 54(12), 2678-2688.

[8] Kaernbach, C. (2001). Adaptive threshold estimation with unforced-choice tasks. Perception \& Psychophysics, 2001, 63, 1377-1388.

[9] Tan, H. Z., Durlach, N. I., Beauregard, G.L., \& Srinivasan, M. (1995). Manual discrimination of compliance using active pinch grasp: The roles of force and work cues. Perception and Psychophysics, 57(4), 495-510.

[10] Jones, L.A. \& Hunter, I.W. (1993). A perceptual analysis of viscosity. Experimental Brain Research, 94(2), 343351. 